\title{
Perfil Biofisico Fetal na Ruptura Prematura das Membranas
}

\author{
Fetal Biophysical Profile in Premature Rupture of the Membranes \\ Vardeli Alves de Moraes ${ }^{1}$, Luis Kulay $\mathrm{Jr}^{2}$, Anna Maria Bertini ${ }^{2}$
}

\begin{abstract}
RESUM0
Objetivo: avaliar as modificações decorrentes da ruptura prematura das membranas sobre as variáveis do perfil biofisico fetal comparando-as às encontradas em gestantes com membranas integras. No grupo com ruptura prematura das membranas foi analisada, ainda, a associação das variáveis biofisicas com os índices de Apgar no $1^{\circ}$ e $5^{\circ}$ minutos e com a presença ou não de corioamnionite clínica e infecção neonatal.

Pacientes e Métodos: em estudo prospectivo foram realizados 112 perfis biofisicos fetais em 60 gestantes com ruptura prematura das membranas entre a $28^{a}$ e a $40^{a}$ semana de gestação, sendo que apenas o último perfil biofisico fetal foi analisado e comparado com 60 perfis biofisicos fetais de gestantes com idades gestacionais idênticas às do grupo estudo e com membranas integras.

Resultados: a análise estatística revelou que a ruptura prematura das membranas diminuiu a reatividade fetal, não interferiu nos movimentos corpóreos fetais, diminuiu a freqüência dos movimentos respiratórios fetais, não determinou modificações no tônus fetal e diminuiu consideravelmente o indice de líquido amniótico. Quanto à predição de corioamnionite e infecção neonatal, o perfil biofisico fetal não demonstrou ter validade estatisticamente significativa; no entanto, quando as variáveis biofisicas estavam presentes, ficou demonstrada de forma evidente a correlação com a ausência de corioamnionite e infecção neonatal. O resultado do último perfil biofisico fetal associou-se significativamente com o indice de Apgar no $5^{\circ}$ minuto.

Conclusão: o perfil biofisico fetal deve ser utilizado rotineiramente em gestantes com ruptura prematura das membranas com o propósito de se avaliar a vitalidade fetal e para se detectarem os fetos com menor probabilidade de infecção, principalmente aqueles com idades gestacionais abaixo da $34^{a}$ semana, nos quais se toma a conduta conservadora.
\end{abstract}

PALAVRAS CHAVE: Vitalidade fetal. Perfil biofisico fetal. Ruptura prematura das membranas.

\section{Introdução}

A ruptura prematura das membranas (RPM) é uma intercorrência obstétrica cuja incidência varia de acordo com a população estudada e com os fatores de risco, apresentando alta incidência no período pré-termo representada por $41,67 \%$ dos casos de RPM estudados por Moraes $^{1,2}$. A RPM acarreta várias complicações

${ }^{1}$ Departamento de Ginecologia e Obstetrícia da Faculdade de Medicina da Universidade Federal de Goiás.

${ }^{2}$ Disciplina de Obstetrícia da Universidade Federal de São Paulo

- Escola Paulista de Medicina.

Correspondência:

Vardeli Alves de Moraes

Rua T - $48 \mathrm{n}^{\circ} .686$ apartamento 101 - setor Bueno

74210-190 - Goiânia - Goiás

Fax: (62) 225-9193 maternas e fetais imediatas, como a prematuridade, infecção, hipoplasia pulmonar, sofrimento fetal intraparto, endometrite e infecção neonatal. Nos casos em que o parto ocorre antes da 34a semana de gestação, eleva-se a incidência da morbidade e mortalidade perinatal decorrentes do desconforto respiratório, hemorragia intraventricular, retinopatia e paralisia cerebral ${ }^{3}$.

Pelo fato de as complicações resultantes da prematuridade serem mais freqüentes do que a septicemia neonatal como fonte de morbidade e mortalidade perinatal, há crescente tendência na atualidade em se adotar a conduta conservadora nas gestantes com RPM antes da $34^{\mathrm{a}}$ sema$\mathrm{na}^{4}$, propondo-se prolongar a gestação na expectativa de que a maturidade pulmonar seja atingida, aumentando com isso a sobrevida pós-na- 
tal.

A real dificuldade na conduta se deve às gestantes que têm RPM antes da 34aㅗ semana de gestação sem infecção clínica, nas quais a conduta conservadora é tomada. Nestes casos, o grande dilema é determinar a duração do período de latência sem aumentar substancialmente os riscos tanto maternos como fetais.

Assim, considerações na conduta de pacientes com RPM devem necessariamente incluir cuidadosa avaliação fetal para diminuir o desenvolvimento dessas potenciais complicações e indicar a antecipação do parto antes que elas ocorram. Manning ${ }^{5}$ enfatiza que a crescente habilidade para catalogar acuradamente a resposta fetal aos possiveis efeitos deletérios da doença materna aumentou o nosso entendimento de como as condições maternas influenciam a saúde fetal, tornando possivel identificar e diferenciar o risco fetal do risco materno. Isto permite ao perinatologista abandonar uma conduta universal ou genérica e adotar o conceito de um teste de avaliação da vitalidade fetal específico à doença materna, recomendando que este conceito de teste específico para a doença passe a ser o formato padrão para os testes anteparto de avaliação da vitalidade fetal. Assim Vintzileos ${ }^{6}$, seguindo esta orientação, indica que os testes específicos para as pacientes com RPM são a cardiotocografia (CTG), o volume de liquido amniótico e o perfil biofísico fetal $(\mathrm{PBF})$.

Na RPM o perfil biofísico fetal reveste-se de importância devido às complicações inerentes a ela já citadas anteriormente, sendo que a mais importante é a identificação do feto de risco para desenvolver septicemia neonatal. Esta identificação pode não depender apenas do desenvolvimento de corioamnionite clínica.

Pelas razões inicialmente anotadas e pela necessidade de identificação precoce das complicações infecciosas advindas da RPM, é relevante acompanhar a vitalidade destes fetos por meio da avaliação das variáveis biofísicas. Esta avaliação, além de detectar os fetos de risco para infecção, identifica também os fetos em sofrimento que necessitam antecipação do parto, bem como aqueles que podem permanecer intra-útero com segurança.

A RPM é conceituada como sendo a ruptura das membranas ovulares, resultando em saída espontânea de líquido amniótico através do colo uterino e cavidade vaginal, exteriorizandose até a vulva e meio externo, antes do início do trabalho de parto. Este conceito independe da idade gestacional e do período de latência, podendo ocorrer em qualquer período da gestação e em tempo variável em relação ao início do trabalho de parto.

O perfil biofísico fetal é a análise de múltiplas variáveis biofísicas fetais combinadas com o objetivo de avaliar a vitalidade fetal. Para obtêlo é necessário realizar a CTG e a ultra-sonografia dinâmica para avaliar a reatividade cardiaca fetal, volume de liquido amniótico, movimentos respiratórios fetais, movimentos corpóreos fetais e o tônus fetal. Os primeiros trabalhos que utilizaram o PBF como método específico para avaliar a vitalidade fetal em gestantes com RPM foram os de Vintzileos et al. ${ }^{7}$, verificando que um PBF anormal pode ser um preditor precoce de infecção fetal e que os primeiros sinais de infecção fetal foram a cardiotocografia não-reativa e a ausência de movimentos respiratórios fetais. Já a ausência de movimentos corpóreos fetais e a perda do tônus fetal foram sinais tardios de infecção.

Por sua vez, Kivikoski et al. ${ }^{8}$, avaliando 44 gestantes com RPM, sem corioamnionite clinica, observaram que em $65 \%$ dos fetos ocorriam movimentos respiratórios e no grupo controle (sem RPM) encontraram estes movimentos, em 90\% dos casos. Isto demonstra que as modificações no ambiente intra-útero, decorrentes da RPM, são suficientes para alterar a freqüência dos movimentos respiratórios fetais mesmo na ausência de infecção.

O valor do PBF em paciente com RPM foi também testado por Roussis et al. ${ }^{9}$, que o realizaram diariamente. O resultado da gestação refletido pelo desenvolvimento de corioamnionite clínica e/ou septicemia neonatal foi correlacionado com os componentes individuais do PBF. A melhor sensibilidade foi obtida pela ausência de movimentos respiratórios fetais (94\%). A mais alta especificidade foi obtida pela ausência de movimentos corpóreos fetais $(100 \%)$ e a combinação de ausência de movimentos corpóreos fetais e de movimentos respiratórios fetais (100\%). Inferiram, assim, com seus resultados que, diante de RPM, o parto deve ser considerado para a paciente com ausência de movimentos respiratórios e com uma CTG que mudou de reativa para não-reativa.

Propusemo-nos a avaliar as modificações decorrentes da ruptura prematura das membranas sobre as variáveis do perfil biofísico fetal, comparando-as às encontradas em pacientes com membranas integras. Intentamos, ainda, analisar, nas gestantes com ruptura prematura das membranas, a associação das variáveis biofisicas com os índices de Apgar no 1o e 5o minutos e com a presença ou não de corioamnionite clínica e infecção neonatal. 


\section{Pacientes e Métodos}

Para avaliarmos prospectivamente as modificações nas variáveis biofísicas do PBF resultantes da abrupta perda de líquido amniótico, foram realizados 112 perfis biofísicos fetais em 60 gestantes com RPM entre a $28^{\text {a }}$ e a $40^{\text {a }}$ semanas de gestação, sendo que, para comparação com o grupo controle e para a avaliação das variáveis biofísicas, utilizamos apenas o último PBF, totalizando então 60 perfis, o que constitui o grupo estudo. O grupo controle, por sua vez, foi representado por 60 perfis biofísicos fetais obtidos de gestantes normais com membranas integras. Para avaliarmos a associação das variáveis do PBF com os índices de Apgar no 1으 e 5o minutos e com a presença ou não de corioamnionite clínica e infecção neonatal, analisamos, também, o resultado do último perfil biofísico fetal das gestantes do grupo estudo. As pacientes do grupo estudo mantiveram-se internadas até o dia do parto, sem inibição da contratilidade uterina, sem antibioticoprofilaxia e utilizando corticoterapia no grupo de idade gestacional entre 28 e 34 semanas. Pretendendo rastrear a corioamnionite, solicitamos tomada da temperatura corporal e pulso materno a cada 4 horas, bem como hemograma a cada dois dias. O toque vaginal foi proscrito até a fase ativa do trabalho de parto e a cesariana somente foi realizada quando havia indicação obstétrica. A cardiotocografia foi sempre realizada antes da ultra-sonografia, com a paciente em decúbito lateral, durante 20 minutos. Decorrido este tempo, se o feto não apresentasse acelerações transitórias ou periódicas, era realizada a estimulação vibroacústica durante 3 segundos sobre o abdome materno junto ao polo cefálico. Os cardiotocogramas foram classificados como apresentando padrão normal e padrão patológico de acordo com critérios estabelecidos por Oliveira et al. ${ }^{10}$.

$\mathrm{O}$ volume de líquido amniótico presente na cavidade uterina foi determinado pelo índice de líquido amniótico (ILA) utilizando a técnica descrita por Phelan et al. ${ }^{11} \mathrm{e}$ interpretado pelo método descrito por Moore e Cayle ${ }^{12}$. Os movimentos respiratórios fetais foram analisados por ultrasonografia em tempo real, pela observação do movimento torácico paradoxal na inspiração, isto é, depressão da parede torácica e expansão da parede abdominal ${ }^{13}$. Os movimentos corpóreos fetais foram inicialmente analisados pela CTG, que os registra sob a forma de pequenas e múltiplas elevações verificadas na parte inferior do traçado do cardiotocograma. Estes movimentos em fetos hígidos associam-se a acelerações tran- sitórias; portanto, a presença de acelerações transitórias e/ou as elevações descritas já caracterizam a presença de movimentos corpóreos fetais no PBF. Na ausência destes movimentos na CTG, a presença ou a ausência dos mesmos foi confirmada pela ultra-sonografia dinâmica, observando movimentos do tronco, polo cefálico e membros. O tônus fetal foi avaliado inicialmente pela presença dos movimentos corpóreos fetais no cardiotocograma, uma vez que partimos do princípio de que somente há movimento fetal se existe tônus fetal; portanto, diante de qualquer movimento corporal e de acelerações transitórias considerou-se que o feto apresentava tônus normal.

$\mathrm{Na}$ ausência de movimento corpóreo fetal e de acelerações transitórias no cardiotocograma, a presença ou ausência de tônus fetal foi confirmada pela ultra-sonografia em tempo real, observando-se a presença ou não de episódios de quaisquer movimentos.

Após a avaliação individual de cada atividade biofísica fetal, elas foram agrupadas em 5 grupos, da seguinte forma: grupo I - CTG normal, movimentos respiratórios presentes, independente do ILA; grupo II - CTG normal, movimentos respiratórios fetais ausentes, independente do ILA; grupo III - CTG de padrão patológico, movimentos respiratórios presentes, independente do ILA; grupo IV - CTG de padrão patológico, movimentos respiratórios ausentes e ILA acima do 5o percentil para a idade gestacional; grupo V - CTG de padrão patológico, movimentos respiratórios ausentes e ILA abaixo do 5응 percentil para a idade gestacional.

Utilizamos critérios modificados do perfil biofisico fetal original de Manning et al. ${ }^{13}$ porque a utilização de todos os parâmetros da CTG basal e estimulada, ao invés de apenas a presença ou não de acelerações aos movimentos fetais, tem trazido mais subsídios do que um parâmetro único. A utilização do índice de líquido amniótico nos quatro quadrantes correlacionado à idade gestacional também nos têm fornecido resultados mais fidedignos do que a medida do maior diâmetro vertical de um único bolsão de líquido amniótico. Finalmente, porque a presença de qualquer movimento fetal à cardiotocografia fetal basal e/ou estimulada já caracteriza sinal de normalidade tanto para o parâmetro movimento fetal quanto para o tônus fetal.

$\mathrm{Na}$ comparação das variáveis individuais do PBF entre o grupo estudo e o grupo controle, para análise das tabelas de contingências, utilizamos os testes de $\chi^{2}$, teste exato de Fisher e teste U de Mann-Whitney quando apropriados a cada tabela. A análise da variância de Kruskall- 
Wallis foi utilizada para analisar a correlação entre o resultado do último $\mathrm{PBF}$ com os índices de Apgar e com a incidência de corioamnionite clínica e infecção neonatal no grupo estudo. O nível de rejeição da hipótese de nulidade (Ho) foi considerado de $5 \%(p<0.05)$.

\section{Resultados}

A corioamnionite esteve presente em 11,7\% dos casos e a infecção neonatal em 8,3\%. O parto normal ocorreu em $66,7 \%$ e a cesariana foi indicada em 33,3\%. A insuficiência respiratória aguda decorrente da prematuridade ocorreu em $6,7 \%$.

Observamos diminuição da variabilidade da freqüência cardíaca fetal em gestantes com RPM quando comparadas com o grupo controle. Não encontramos nenhum caso de variabilidade menor que $5 \mathrm{bpm}$ no grupo controle, ao passo que no grupo estudo a detectamos em 10\% dos registros.

Os casos de RPM cursaram com maior incidência de desacelerações variáveis (15\%) do que as gestantes com membranas integras (1,7\%). Nos casos de RPM, as acelerações transitórias ocorreram em menor número $(83,3 \%)$ do que no grupo controle (95\%). Evidencia-se, com significância estatística importante, que nas gestantes com RPM há maior incidência de cardiotocogramas classificados dentro do padrão patológico (Tabela 1).

Tabela 1 - Classificação do cardiotocograma quanto ao padrão normal ou patológico em gestantes com RPM, comparadas com as do grupo controle.

\begin{tabular}{lrrrc}
\hline & & \multicolumn{2}{c}{ Grupo } \\
Cardiotocografia & & RPM & & \multicolumn{2}{c}{ Controle } \\
\hline & $\mathbf{n}$ & $\%$ & $\mathbf{n}$ & $\%$ \\
Padrão normal & 51 & 85 & 60 & 100 \\
Padrão patológico & 9 & 15 & 0 & - \\
Total & 60 & 100 & 60 & 100 \\
\hline
\end{tabular}

$p=0,001$ pelo teste exato de $=$ Fisher

Não houve diferença estatisticamente significativa entre pacientes com RPM e o grupo controle quanto à presença ou ausência de movimentos corpóreos fetais.

Em relação aos movimentos respiratórios fetais, ficou demonstrado, de forma evidente, que em gestantes com ruptura prematura das membranas houve diminuição dos movimentos respiratórios fetais (Tabela 2). Observamos que tanto no grupo de gestantes com RPM quanto no grupo controle não houve alterações no tônus fetal.

\begin{tabular}{|c|c|c|c|c|}
\hline \multirow{3}{*}{$\begin{array}{l}\text { Movimentos } \\
\text { respiratórios fetais }\end{array}$} & \multicolumn{4}{|c|}{ Grupo } \\
\hline & \multicolumn{2}{|c|}{ RPM } & \multicolumn{2}{|c|}{ Controle } \\
\hline & $\mathrm{n}$ & $\%$ & $\mathrm{n}$ & $\%$ \\
\hline Presentes & 32 & 53,4 & 52 & 86,7 \\
\hline Ausentes & 28 & 46,6 & 8 & 13,3 \\
\hline Total & 60 & 100,0 & 60 & 100,0 \\
\hline
\end{tabular}

Ficou demonstrado também que o indice de líquido amniótico estava bastante diminuído em gestantes com RPM, caracterizando o oligoidrâmnio, quando comparado com o do grupo controle (Tabela 3). Quando o PBF foi classificado de acordo com as atividades biofísicas fetais combinadas com o ILA em gestantes com RPM e comparado com o do grupo controle, notamos que em relação ao grupo controle, a ruptura prematura das membranas modificou significativamente as variáveis biofisicas fetais e o ILA (Tabela 4).

Tabela 3 - Índice de líquido amniótico (ILA) em gestantes com RPM comparadas com as do grupo controle.

\begin{tabular}{lrrrr}
\hline & & & Grupo & \\
ILA (percentil) & & RPM & & \multicolumn{2}{c}{ Controle } \\
& $\mathbf{n}$ & $\%$ & $\mathbf{n}$ & $\%$ \\
\hline$<2,5$ & 45 & 75,0 & 0 & - \\
$2,5-5$ & 6 & 10,0 & 0 & - \\
$5-10$ & 9 & 15,0 & 17 & 28,3 \\
$50-95$ & 0 & - & 43 & 71,7 \\
Total & 60 & 100,0 & 60 & 100,0 \\
\hline $\mathrm{p}=0,000$ pelo teste Ud de Mann-Whitney & & &
\end{tabular}

Tabela 4 - Classificação do perfil biofísico fetal de acordo com as atividades biofísicas fetais combinadas com o índice de líquido amniótico em gestantes com RPM, comparadas com as do grupo controle.

\begin{tabular}{lrrrr}
\hline & & & Grupo & \\
Classificação & & RPM & & \multicolumn{2}{c}{ Controle } \\
& $\mathbf{n}$ & $\%$ & $\mathbf{n}$ & $\%$ \\
\hline Grupo I * & 25 & 41,7 & 52 & 86,7 \\
Grupo II & 26 & 43,3 & 8 & 13,3 \\
Grupo III & 5 & 8,3 & 0 & - \\
Grupo IV & 1 & 1,7 & 0 & - \\
Grupo V & 3 & 5,0 & 0 & - \\
Total & 60 & 100,0 & 60 & 100,0 \\
\hline $\begin{array}{l}\text { p=0,000 pelo teste Ude Mann-Whitney } \\
\text { *definicão dos grupos-ver pág.202 }\end{array}$ & & &
\end{tabular}

* definição dos grupos - ver pág. 202 
A presença de movimentos respiratórios fetais teve melhor correlação com a ausência de corioamnionite do que a ausência dos mesmos em relação à presença de infecção, embora sem significância estatística. O mesmo ocorreu com a relação entre movimentos respiratórios fetais e infecção neonatal.

Não houve associação estatisticamente significativa entre a presença ou ausência dos movimentos corpóreos fetais e a presença ou ausência de corioamnionite, mas em relação à infecção neonatal, houve correlação (Tabela 5). Os poucos casos de corioamnionite (7 casos) e de infecção neonatal (5 casos) somente ocorreram nos casos com oligoidrâmnio e em nenhum caso com ILA acima do $5^{\circ}$ percentil para a idade gestacional.

Tabela 5 - Presença ou ausência de movimentos corpóreos fetais (MCF) associados com a presença ou não de infecção neonatal.

\begin{tabular}{lccrc}
\hline \multirow{2}{*}{ MCF } & \multicolumn{3}{c}{ Infecção neonatal } \\
& $\mathbf{n}$ & $\%$ & $\mathbf{n}$ & $\%$ \\
\hline Presentes & 2 & 40,0 & 49 & 89,1 \\
Ausentes & 3 & 60,0 & 6 & 10,9 \\
Total & 5 & 100,0 & 55 & 100,0 \\
\hline
\end{tabular}

$p=0,02$ pelo teste exato de Fisher

Sensibilidade $=60 \%$

Especificidade $=89,1 \%$

Valor preditivo positivo $=33,3 \%$

Valor preditivo negativo $=96,0 \%$

Não houve associação estatística entre o resultado da CTG com a presença ou ausência de corioamnionite; no entanto, em relação à infecção neonatal observamos associação positiva (Tabela 6). Não houve correlação significante entre a classificação do PBF em grupos de atividades biofisicas com corioamnionite, o mesmo ocorrendo com a infecção neonatal.

Tabela 6 - Correlação da cardiotocografia (CTG) com a presença ou ausência de infecção neonatal.

\begin{tabular}{lccrr}
\hline & \multicolumn{3}{c}{ Infecção neonatal } \\
CTG & \multicolumn{2}{c}{ Presente } & \multicolumn{2}{c}{ Ausente } \\
& $\mathbf{n}$ & $\%$ & $\mathbf{n}$ & $\%$ \\
\hline Padrão normal & 2 & 40,0 & 49 & 89,0 \\
Padrão patológico & 3 & 60,0 & 6 & 11,0 \\
Total & 5 & 100,0 & 55 & 100,0 \\
\hline p=0,02 pelo teste exato de Fisher & & & & \\
Sensibilidade $=60 \%$ & & & & \\
Especificidade $=90,71 \%$ & & & & \\
Valor preditivo positivo $=33,3 \%$ & & & & \\
Valor preditivo negativo $=96,1 \%$ & & &
\end{tabular}

Quando o índice de Apgar no 1ำ minuto foi correlacionado com a classificação do PBF em grupos de atividades biofísicas, não observamos associação estatística, sendo que houve associação positiva com o índice de Apgar no 5o minuto (Tabela 7).

Tabela 7 - Índice de APGAR no $5^{\circ}$. minuto correlacionado com a classificação do PBF quanto ao grupo.

\begin{tabular}{|c|c|c|c|c|c|c|c|c|c|c|}
\hline \multirow[b]{3}{*}{ Apgar } & \multicolumn{10}{|c|}{ Perfil biofísico fetal } \\
\hline & \multicolumn{2}{|c|}{ Grupo I * } & \multicolumn{2}{|c|}{ Grupo II } & \multicolumn{2}{|c|}{ Grupo III } & \multicolumn{2}{|c|}{ Grupo IV } & \multicolumn{2}{|c|}{ Grupo V } \\
\hline & $\mathrm{n}$ & $\%$ & $\mathrm{n}$ & $\%$ & $\mathrm{n}$ & $\%$ & $\mathrm{n}$ & $\%$ & $\mathrm{n}$ & $\%$ \\
\hline$<7$ & 2 & 8,0 & 1 & 3,8 & 0 & - & 0 & - & 2 & 66,7 \\
\hline$=>7$ & 23 & 92,0 & 25 & 96,2 & 5 & 100,0 & 5 & 100,0 & 1 & 33,3 \\
\hline Total & 25 & 100,0 & 26 & 100,0 & 5 & 100,0 & 5 & 100,0 & 3 & 100,0 \\
\hline
\end{tabular}

Não houve correlação estatística entre índice de Apgar no 1 minuto e a CTG. O indice de Apgar no 5o minuto correlacionou-se positivamente com o resultado do último cardiotocograma, demonstrando que, quando normal, associou-se mais freqüentemente com vitalidade preservada do que o padrão patológico com feto comprometido (Tabela 8). Não houve correlação estatística entre o índice de Apgar no $1^{\circ}$ e $5^{\circ}$ minutos com o ILA.

Tabela 8 - Índice de APGAR no $5^{\circ}$. minuto correlacionado à cardiotocografia.

\begin{tabular}{lcccc}
\hline & \multicolumn{4}{c}{ Cardiotocograma } \\
Apgar & $\mathbf{n}$ & $\%$ & Padrão normal & patológico \\
\% & 3 & 5,9 & 3 & 33,3 \\
\hline$<7$ & 48 & 94,1 & 6 & 66,7 \\
$=>7$ & 51 & 100,0 & 9 & 100,0 \\
\hline
\end{tabular}

$p=0,038$ pelo teste exato de Fisher

Sensibilidade $=50 \%$

Especificidade $=88,9 \%$

Valor preditivo positivo $=33,3 \%$

Valor preditivo negativo $=94,1 \%$

\section{Discussão}

Os nossos resultados demonstraram modificações nas variáveis biofísicas fetais, decorrentes do oligoidrâmnio subitamente instalado em gestantes que apresentam ruptura prematura das membranas. Dentre elas, verificamos diminuição na variabilidade da freqüência cardíaca fetal basal. Estes dados são concordantes com os de Sarno Jr et al. ${ }^{14}$. 
Com referência às desacelerações variáveis, os dados demonstram indubitavelmente que, quando ocorre a RPM, aumenta a ocorrência destas desacelerações, quando comparadas com gestantes sem RPM. Vários autores afirmam que o oligoidrâmnio abruptamente instalado nas gestantes com RPM propicia o surgimento de desacelerações variáveis ${ }^{1,7,14,15}$. Estas desacelerações surgem em decorrência da compressão do cordão umbilical, resultante da diminuição do volume de líquido amniótico, e estão associadas ao grau de oligoidrâmnio.

Houve menor número de gestantes com acelerações transitórias dos movimentos corpóreos fetais do que nas gestantes normais. Como a presença destas acelerações representa o parâmetro mais importante para afirmar a reatividade fetal, os resultados apresentados mostram que, nas gestantes com RPM, há diminuição da reatividade fetal. Estes resultados divergem de Vintzileos et al. ${ }^{16}$, que referem incidência aumentada de CTG reativa em pacientes com RPM, e são concordantes com os de Sarno $\mathrm{Jr}$ et al. ${ }^{14}$ e Harding et al. ${ }^{17}$. Esta divergência com os resultados de Vintzileos é, provavelmente, dependente do maior ou menor grau de oligoidrâmnio presente nos grupos distintos de pacientes. É importante assinalar que, no presente estudo, 85\% dos casos apresentavam ILA abaixo do 5o percentil para a idade gestacional, sendo que em $75 \%$ dos casos, o ILA esteve abaixo do percentil 2,5 .

Em relação à classificação dos cardiotocogramas, notamos que no grupo de gestantes com RPM há maior incidência de CTG compativel com padrão patológico, evidenciando que a RPM determina diminuição da reatividade cardiaca fetal e comprometimento da vitalidade fetal. Quanto aos movimentos respiratórios fetais, detectamos que nas gestantes com RPM há diminuição significativa destes movimentos. A diminuição dos movimentos respiratórios já foi relatada por vários autores ${ }^{7,18}$. Estes explicam que, na presença de reduzido volume de líquido amniótico, os movimentos respiratórios diminuem como resultado da compressão do tórax fetal. Os nossos dados confirmam que na avaliação da vitalidade fetal em pacientes com RPM, a atenção deve ser dada não ao escore total do PBF proposto por Manning et al. ${ }^{13}$ e sim aos componentes individuais e à combinação deles para cada feto; desta forma, classificamos os perfis biofísicos em 5 grupos já expostos e observamos que no grupo estudo, apesar de ter havido predominância de casos nos grupos I e II, houve casos em todos os grupos. No grupo controle foram observados apenas casos complicados no grupo I e no grupo II.
Estes dados confirmam que a ruptura prematura das membranas modifica as variáveis biofisicas fetais. A correlação entre a presença ou ausência dos movimentos corpóreos fetais com a presença ou não de infecção neonatal demonstrou que a ausência destes movimentos associou-se com infecção neonatal, sendo que a presença deles associou-se com a ausência de infecção neonatal. Estes dados são corroborados por Goldstein et al. ${ }^{19}$, os quais afirmam que a presença de um episódio de movimento corpóreo fetal foi associada com ausência de infecção intra-amniótica em $100 \%$ dos casos. Por outro lado, a ausência de movimentos fetais associouse com cultura positiva de liquido amniótico em todos os casos. Postulam, portanto, que uma possivel explicação para este fato é que a interleucina-1 produzida pelo feto, em resposta à agressão bacteriana, pode produzir alterações nas atividades corporais fetais. Os dados de Roussis et al. ${ }^{9}$ também confirmam os nossos resultados, pois os autores verificaram que a ausência de movimentos corpóreos fetais foi bastante específica para a presença de corioamnionite e infecção neonatal, com valor preditivo positivo de $100 \%$.

Observa-se portanto que houve associação entre a cardiotocografia anteparto e a infecção neonatal em pacientes com RPM, apresentando sensibilidade de $60 \%$ e especificidade de 90,7\%. Estes resultados são concordantes com os de Vintzileos et al. ${ }^{7}$, que encontraram $30,1 \%$ de infecção neonatal em sua casuística e afirmaram que os primeiros sinais de infecção neonatal foram a cardiotocografia não-reativa e a ausência de movimentos respiratórios fetais.

Com o propósito de avaliar a validade da classificação do PBF quanto à combinação das variáveis biofísicas fetais em informar as condições fetais intra-útero e ao nascimento, fizemos a associação dos grupos já citados anteriormente com o indice de Apgar no 1o e no 5o minuto. Não se notou nenhuma correlação estatística entre o índice de Apgar no 1을 minuto e a combinação das variáveis biofísicas. Resultado diferente ocorreu com o índice de Apgar no 5o minuto, em que observamos que houve correlação estatisticamente significativa, mostrando que a combinação das variáveis biofísicas do PBF é útil para predizer a vitalidade fetal intra-útero, sendo que, à medida em que elas vão se deteriorando, diminuem os índices de Apgar ao 5o minuto após o nascimento.

Os indices de Apgar foram, ainda, associados com a cardiotocografia anteparto isolada do PBF e verificamos que não houve correlação estatística entre o resultado da CTG e o índice de 
Apgar no 1ำ minuto e, sim, com o índice de Apgar no 5o minuto. Estes resultados confirmam os de Moraes $^{1}$, que verificou associação altamente significativa entre o resultado da última CTG com indices de Apgar no 5o minuto de nascimento. Foi observado um valor preditivo negativo bem superior ao valor preditivo positivo, evidenciando que a CTG normal tem maior validade em demonstrar o não-comprometimento fetal do que a cardiotocografia de padrão patológico em mostrar vitalidade fetal comprometida.

Finalizando as nossas discussões, podemos constatar que o oligoidrâmnio abruptamente instalado nas gestantes com RPM impõe condições adversas ao feto, as quais são detectadas mais pelas variáveis biofísicas isoladas ou combinadas do que pelo escore total do perfil biofísico fetal e que, apesar de o perfil biofísico fetal proposto por Manning et al. ${ }^{13}$ ser criticado na atualidade, porque agrupa vários componentes biofísicos que não têm o mesmo poder de predição do comprometimento fetal por haverem ocorrido várias modificações nos parâmetros por eles propostos, acreditamos que a avaliação das atividades biofísicas fetais consagradas pelo uso com o nome de perfil biofísico fetal, seja ele completo ou simplificado, ainda deve continuar a fazer parte da propedêutica da vitalidade fetal. Concluimos que o perfil biofísico fetal mostrou ser propedêutica adequada para a avaliação da vitalidade fetal em gestantes com RPM, sendo observado que ela determina modificações nas variáveis biofísicas fetais, sobretudo no indice de líquido amniótico, movimentos respiratórios fetais e CTG basal, quando comparadas com as das gestantes normais. Os resultados mostraram, ainda, que a análise das variáveis biofísicas combinadas ou individualmente é mais eficaz em avaliar a vitalidade fetal em gestantes com RPM do que o escore total. Quanto à predição de corioamnionite e infecção neonatal, o perfil biofísico fetal não demonstrou ter validade estatisticamente significativa; no entanto, quando as variáveis biofísicas estavam presentes, ficou demonstrada de forma evidente a correlação com a ausência de corioamnionite e infecção neonatal, permitindo, assim, assumir a conduta conservadora diante de casos de RPM em fetos imaturos. As variáveis biofísicas que mais correlacionaram-se com a infecção neonatal foram o oligoidrâmnio, a cardiotocografia basal e os movimentos corpóreos fetais. Na correlação entre as variáveis biofísicas combinadas, conforme os vários grupos por nós propostos, mostrou-se que o PBF é útil para predizer a vitalidade fetal em pacientes com RPM, destacando-se a cardiotocografia e o indice de líquido amniótico.

\section{SUMMARY}

Purpose: to evaluate the modifications due to premature rupture of the membranes of variables of the fetal biophysical profile, comparing them to the ones found in pregnant women with intact membranes. In the group with premature rupture of the membranes, the association of biophysical variables with the Apgar score at the first and fifth minutes and with the occurrence or not of clinical chorioamnionitis and neonatal infection was analyzed. Patients and Methods: in a prospective study, 112 fetal biophysical profiles were established in 60 pregnant women with premature rupture of the membranes at a period between the 28th and the 40th week of pregnancy, and only the last fetal biophysical profile was analyzed and compared to other 60 fetal biophysical profiles of pregnant women with gestational ages identical to the group of study and with intact membranes.

Results: statistical analysis of the results revealed that the premature rupture of the membranes diminished the reactivity, not interfering with the body movements of the fetus. It also diminished the frequency of the respiratory movements of the fetus, not determining modifications of the fetal tonus, and considerably decreasing the amniotic fluid index. In terms of prediction of chorioamnionitis and neonatal infection, the fetal biophysical profile did not show any significant statistic validity; however, when the biophysical variables were present, the correlation with the absence of chorioamnionitis and neonatal infection was clearly shown. The result of the last fetal biophysical profile was strongly associated with the Apgar score at the 5th minute.

Conclusion: the fetal biophysical profile should be used routinely in pregnant women with premature rupture of the membranes with the purpose of evaluating fetal vitality, and in order to detect those fetuses at a higher risk of infection, specially those with gestational ages of less than 34 weeks. Regarding those fetuses it is best to use a conservative approach.

KEY WORDS: Fetal vitality. Fetal biophysical profile. Premature rupture of the membranes.

\section{Referências}

1. Moraes VA. A cardiotocografia anteparto em gestantes com ruptura prematura de membranas. [Dissertação]. São Paulo: Universidade Federal de São Paulo; 1993.

2. Moraes VA. Perfil biofisico fetal na ruptura prematura das membranas. [Tese]. São Paulo: Universidade Federal de São Paulo; 1999. 
3. Vintzileos AM, Campbell WA, Rodis JF. Antepartum surveillance in patients with preterm premature rupture of the membranes. Clin Obstet Gynecol 1991; 34:779-93.

4. Bertini AM, Taborda, W. Ruptura prematura das membranas. Femina 1997; 25:723-34.

5. Manning FA. Dynamic ultrasound-based fetal assessment: the fetal biophysical profile score. Clin Obstet Gynecol 1995; 38:26-44.

6. Vintzileos AM. Antepartum fetal surveillance. Clin Obstet Gynecol 1995; 38:1-2.

7. Vintzileos AM, Campbell WA, Nochimson DJ, Connoly ME, Fuenfer MM, Hoehn GJ. The fetal biophysical profile in patients with premature rupture of the membranes - an early predictor of fetal infection. Am J Obstet Gynecol 1985; 152:510-6.

8. Kivikoski AI, Amon E, Vaalamo PO, Pirhonen J, Kopta MM. Effect of third-trimester premature rupture of membranes on fetal breathing movements: a prospective case-control study. Am J Obstet Gynecol 1988; 159:1474-7.

9. Roussis P, Rosemond RL, Glass C, Boehm FH. Preterm premature rupture of membranes: detection of infection. Am J Obstet Gynecol 1991; 165:1099-104.

10. Oliveira AM, Souza JB, Santana RM, et al. Contribuição ao estudo da cardiotocografia prenatal. O teste sem estresse. Rev Bras Ginecol Obstet 1983; 5:150-6.

11. Phelan JP, Smith CV, Broussard P, Small M. Amniotic fluid volume assessment with the fourquadrant technique at 36-42 weeks' gestation. J Reprod Med 1987; 32:540-2.
12. Moore TR, Cayle JE. The amniotic fluid index in normal human pregnancy. Am J Obstet Gynecol 1990; 162:1168-73.

13.Manning FA, Platt LD, Sipos L. Antepartum fetal evaluation: development of a fetal biophysical profile. Am J Obstet Gynecol 1980; 136:787-95.

14.Sarno AP Jr, Ahn MO, Phelan JP. Intrapartum amniotic fluid volume at term. Association of rupture membranes, oligohydramnios and increased fetal risk. J Reprod Med 1990; 35:71923.

15.Alencar Jr CA, Santana RM, Santos JFK, et al. Estudo crítico da utilização da cardiotocografia anteparto em pacientes com ruptura prematura das membranas. Femina 1991; 19:897-9.

16.Vintzileos AM, Feinstein SJ, Lodeiro JG, Campbell WA, Weinbaum PJ, Nochimson DJ. Fetal biophysical profile and the effect of premature rupture of the membranes. Obstet Gynecol 1986; 67:818-23.

17.Harding JA, Jackson DM, Lewis DF, Major CA, Nageotte MP, Asrat T. Correlation of amniotic fluid index and nonstress test in patients with preterm premature rupture of membranes. Am J Obstet Gynecol 1991; 165:1088-94.

18. Roberts AB, Goldstein I, Romero R, Hobbins JC. Comparison of total fetal activity measurement with the biophysical profile in predicting intraamniotic infection in preterm rupture of membranes. Ultrasound Obstet Gynecol 1991; 1:36-9.

19.Goldstein I, Copel JA, Hobbins JC. Fetal behavior in preterm premature rupture of the membranes. Clin Perinatol 1989; 16:735-54.

\section{Portanto, publique!!!}

